MATHEMATICS OF COMPUTATION

Volume 70, Number 234, Pages 525-531

S 0025-5718(00)01195-9

Article electronically published on March 1, 2000

\title{
ON ERROR ESTIMATES \\ FOR GALERKIN SPECTRAL DISCRETIZATIONS OF PARABOLIC PROBLEMS WITH NONSMOOTH INITIAL DATA
}

\author{
JAVIER DE FRUTOS AND RAFAEL MUÑOZ-SOLA
}

\begin{abstract}
We analyze the Legendre and Chebyshev spectral Galerkin semidiscretizations of a one dimensional homogeneous parabolic problem with nonconstant coefficients. We present error estimates for both smooth and nonsmooth data. In the Chebyshev case a limit in the order of approximation is established. On the contrary, in the Legendre case we find an arbitrary high order of convegence.
\end{abstract}

\section{INTRODUCTION}

In this paper we give some results about Galerkin spectral polynomial approximations to a parabolic problem with nonconstant coefficients. We treat both the Chebyshev and the Legendre cases. More precisely, we consider the one-dimensional parabolic problem:

$$
\begin{aligned}
u_{t}-\left(a(x) u_{x}\right)_{x} & =0, & & x \in \Lambda, t \geq 0, \\
u(-1, t)=u(1, t) & =0, & & t \geq 0, \\
u(x, 0) & =u_{0}(x), & & x \in \Lambda,
\end{aligned}
$$

where $\Lambda=(-1,1)$, and $a$ is a smooth function satisfying the classical assumption $0<\underline{a} \leq a(x) \leq \bar{a}$ in $\bar{\Lambda}$, which ensures that the problem is parabolic.

The error estimates which can usually be found in the literature about spectral methods need some regularity hypotheses on the data of the problem. Typically, it is assumed that the solution $u$ of (1) has $m$ square-integrable spatial derivatives in $[0, T]$. With this assumption one is able to get an $\mathcal{O}\left(N^{-m}\right)$ error estimate when a spectral approximation based on polynomials of degree $N$ is used. Some references are, for example, [4] and [3] for the constant coefficient case and [6] for the variable coefficient case. Due to the regularization property of parabolic problems, we can expect that similar estimates will still hold, for positive times, under weaker assumptions on the regularity of the initial condition. For finite element semidiscretizations of (1), error estimates of the form $\mathcal{O}\left(h^{r} t^{-r / 2}\right)$, where $r-1$ denotes the order of the element, have been obtained for rough initial data; see for example [10], [11] and [13]. The negative power of $t$ in the error estimate

Received by the editor January 4, 1999 and, in revised form, April 6, 1999.

2000 Mathematics Subject Classification. Primary 65M70, 65M15.

Key words and phrases. Spectral Galerkin method, parabolic equation, nonsmooth initial data. J. de Frutos was partially supported by project DGICYT PB95-705 and project JCyL VA52/96.

R. Muñoz-Sola was partially supported by project DGICYT PB96-0952. 
is closely related to the lack of differentiability of the solution at $t=0$. We do not know of any analogous results for spectral methods in the literature. The main difference is that the order of convergence in spectral methods is only limited by the regularity of the solution. Then, an $\mathcal{O}\left(N^{-m} t^{-m / 2}\right)$ for any $m$ should be expected when polynomials of degree $N$ are used.

This is indeed the case for the Legendre spectral discretization, i.e., spectral order of convergence is obtained even with no regularity hypothesis on the initial data. On the contrary, this is not true for the Chebyshev discretization. We prove that for nonsmooth data, the order of approximation is limited by $7 / 2$.

The rest of the paper is as follows: In Section 2 we establish our notations and recall the continuous problem and its discretization. In Section 3 we state and prove our negative result for the Chebyshev weight. We restrict ourselves to the constant coefficient case there. Finally, in Section 4 we quote without proof some estimates obtained for the Legendre and Chebyshev discretizations. For the proofs, we refer to 7 .

\section{Preliminares and notations}

2.1. Basic notations. Thorough the paper, we will use the notation

$$
L_{\omega}^{2}=\left\{v:\left.\Lambda \mapsto \mathbb{C}\left|\int_{\Lambda}\right| v\right|^{2} \omega d x<\infty\right\},
$$

where the weight $\omega$ will be $\omega(x)=1$ or $w(x)=\left(1-x^{2}\right)^{-\frac{1}{2}}$ when we deal with the Legendre or the Chebyshev case respectively. The inner product in $L_{\omega}^{2}$ is represented by $(\cdot, \cdot)_{0, \omega}$.

Sobolev spaces of high order with respect to either the Legendre or the Chebyshev weight are denoted by $H_{\omega}^{\nu}, \nu>0$, with norm $\|\cdot\|_{\nu, \omega}$ (see [2] for a precise definition in the Chebyshev case), and

$$
H_{\omega, 0}^{1}=\left\{v \in H_{\omega}^{1}, v(1)=v(-1)=0\right\} .
$$

We denote by $\mathbb{P}^{N}$ the space of the restrictions to $\Lambda$ of the polynomials of degree at most $N$, and define

$$
\mathbb{P}_{0}^{N}=\left\{v \in \mathbb{P}^{N} \mid v( \pm 1)=0\right\} .
$$

2.2. The continuous problem and its discretization. Let $a_{\omega}(\cdot, \cdot)$ be the sesquilinear form over $H_{\omega, 0}^{1}$ defined by

$$
a_{\omega}(u, v)=\int_{\Lambda} a(x) u_{x}(\bar{v} \omega)_{x} d x, \quad \forall u, v \in H_{\omega, 0}^{1} .
$$

Problem (1) can be written in variational form as

$$
\frac{d}{d t}(u(t), \varphi)_{0, \omega}+a_{\omega}(u(t), \varphi)=0, \quad \forall \varphi \in H_{\omega, 0}^{1},
$$

supplemented with the initial condition

$$
u(\cdot, 0)=u_{0}(\cdot) .
$$

The Galerkin spectral discretization has the following variational form:

$$
\frac{d}{d t}\left(u^{N}(t), v^{N}\right)_{0, \omega}+a_{\omega}\left(u^{N}(t), v^{N}\right)=0,
$$


for all $v^{N} \in \mathbb{P}_{0}^{N}$, plus the initial condition

$$
u^{N}(0)=P_{N}\left(u_{0}\right) .
$$

Here, $P_{N}$ stands for the orthogonal projection operator from $L_{\omega}^{2}$ onto $\mathbb{P}_{0}^{N}$.

We denote the error by $e^{N}(t)=u(t)-u^{N}(t)$.

\section{A negative Result for the Chebyshev discretization}

In this section, the weight will be $\omega(x)=\left(1-x^{2}\right)^{-\frac{1}{2}}$, and we restrict our attention to the constant coefficient case, namely $a(x)=1$. In this case, the bilinear form $a_{\omega}(\cdot, \cdot)$ is coercive (see 3]). Hence, problem (3)-(4) is well posed in the Chebyshev norm.

Here, we introduce some notations that will be used later. We denote by $A$ the closed unbounded operator defined by the triplet $\left(V, H, a_{\omega}(\cdot, \cdot)\right)$, where $V=H_{\omega, 0}^{1}$ and $H=L_{\omega}^{2}$ (see [5, Chapter 6]). Since we are now dealing with the particular case $a(x)=1$, we have obviously $D(A)=H_{\omega}^{2} \cap H_{\omega, 0}^{1}$ and $A=-\frac{d^{2}}{d x^{2}}$, considered as defined from $D(A) \subset L_{\omega}^{2} \mapsto L_{\omega}^{2}$. In an analogous way, we define the operator $A_{N}: \mathbb{P}_{0}^{N} \mapsto \mathbb{P}_{0}^{N}$ by

$$
\left(A_{N} v^{N}, \psi^{N}\right)_{0, \omega}=a_{\omega}\left(v^{N}, \psi^{N}\right), \quad \forall \psi^{N} \in \mathbb{P}_{0}^{N},
$$

With this notations, we can write $u(t)=e^{-t A} u_{0}$ and $u^{N}(t)=e^{-t A_{N}} P_{N} u_{0}$. Following [13, we introduce the error operator defined by

$$
E_{N}(t) v=e^{-t A} v-e^{-t A_{N}} P_{N} v, \quad \forall v \in L_{\omega}^{2},
$$

so that $e^{N}(t)=E_{N}(t) u_{0}$.

We shall also need the adjoint $A^{*}$ (resp. $A_{N}^{*}$ ) of the operator $A\left(\right.$ resp. $A_{N}$ ). From [5, Chapter 6], $A^{*}$ is the operator defined by the triplet $\left(H_{\omega, 0}^{1}, L_{\omega}^{2}, a_{\omega}^{*}(\cdot, \cdot)\right)$ with $a_{\omega}^{*}(u, v)=\overline{a_{\omega}(v, u)} \quad \forall u, v \in H_{\omega, 0}^{1}$.

It can be easily seen that

$$
D\left(A^{*}\right)=\left\{v \in H_{\omega, 0}^{1} \mid \frac{1}{\omega} \frac{d^{2}}{d x^{2}}(\omega v) \in L_{\omega}^{2}\right\},
$$

and a simple calculation shows that

$$
A^{*} v=-\frac{1}{\omega} \frac{d^{2}}{d x^{2}}(\omega v)
$$

Note that $D\left(A^{*}\right) \not \subset H_{\omega}^{2}$.

We shall make use the parabolic problem related to $A^{*}$. Corollary 10.6 of [12] Chapter 1] gives us $\left(e^{-t A}\right)^{*}=e^{-t A^{*}}$. Clearly $\left(e^{-t A_{N}}\right)^{*}=e^{-t A_{N}^{*}}$, so that

$$
\left(E_{N}(t)\right)^{*}=e^{-t A^{*}}-i_{N} e^{-t A_{N}^{*}} P_{N} .
$$

Here $i_{N}: \mathbb{P}_{0}^{N} \hookrightarrow L_{\omega}^{2}$ is the canonical injection; note that $i_{N}^{*}=P_{N}$.

It is easy to see that, for any $\phi_{0} \in L_{\omega}^{2}, \phi(t)=e^{-t A^{*}} \phi_{0}$ is the solution of

$$
\frac{d}{d t}(\phi(t), v)_{0, \omega}+a_{\omega}^{*}(\phi(t), v)=0, \quad \forall v \in H_{\omega, 0}^{1},
$$

with initial condition

$$
\phi(0)=\phi_{0}
$$

In the same way, $\phi^{N}(t)=e^{-t A_{N}^{*}} P_{N} \phi_{0}$ is the solution of the spectral Galerkin discretization of (5)-(6). Hence $\left(E_{N}(t)\right)^{*}$ is the associated error operator. 
Now we are able to state our negative result.

Theorem 3.1. Let $a(x)=1, \quad-1<x<1$. Let us suppose that there is a time $t>0$ such that, for any $u_{0} \in L_{\omega}^{2}$, there is a constant $K=K\left(t, u_{0}\right)>0$ with $\left\|e^{N}(t)\right\|_{0, \omega} \leq K\left(t, u_{0}\right) N^{-s}$. Then $s \leq \frac{7}{2}$.

Theorem 3.1 shows that, unlike the finite element case (and the Legendre spectral one), there is a limit on the order of convergence with respect to $N$ which can be reached for any initial data $u_{0} \in L_{\omega}^{2}$, even in the simplest case $a(x)=1$.

Theorems 3.2 and 3.3 are auxiliary results which are needed in the proof of Theorem 3.1. For their proof we refer to [8], [9], where a more general family of spaces related to Jacobi weights is considered.

For $m \in \mathbb{N}$, we define

$$
Z_{\omega}^{m}=\left\{v \in L_{\omega}^{2} \mid\left(1-x^{2}\right)^{\frac{j}{2}} \frac{d^{j} v}{d x^{j}} \in L_{\omega}^{2}, 1 \leq j \leq m\right\}
$$

For $s>0$ arbitrary, $s=m+\sigma$ with $m$ integer and $0<\sigma<1$, we define $Z_{\omega}^{s}$ as

$$
Z_{\omega}^{s}=\left[Z_{\omega}^{m}, Z_{\omega}^{m+1}\right]_{\sigma, 2},
$$

where $[\cdot]_{\sigma, 2}$ stands for the $K$-interpolation method [1].

We have the following characterization of the spaces $Z_{\omega}^{s}$.

Theorem 3.2. Let $s=m+\theta$ be a positive real number with $m$ an integer and $0<\theta<1, s \neq \frac{1}{2}$, and let $a>1$. A norm in $Z_{\omega}^{s}$ which defines this space is

$$
\|u\|=\left[\|u\|_{Z_{\omega}^{m}}^{2}+\iint_{\Delta_{a}} \frac{\left|u^{(m)}(x)-u^{(m)}(y)\right|^{2}}{|x-y|^{1+2 \theta}}\left(1-x^{2}\right)^{s-\frac{1}{2}} d x d y\right]^{\frac{1}{2}},
$$

where the set $\Delta_{a}$ is defined by $\Delta_{a}=\left\{(\xi, \eta) \mid \xi<0, \frac{1+\xi}{a}<1+\eta<a(1+\xi)\right\} \cup$ $\left\{(\xi, \eta) \mid \xi>0, \frac{1-\xi}{a}<1-\eta<a(1-\xi)\right\}$.

The next theorem is an inverse approximation result.

Theorem 3.3. Let $s>0$ and $u \in L_{\omega}^{2}$. If $\forall N \in \mathbb{N}$, there exists $u_{N} \in \mathbb{P}_{N}$ such that $\left\|u-u_{N}\right\|_{0, \omega} \leq B N^{-s}$, with $B=B(u)$ independent of $N$, then $u \in Z_{\omega}^{s-\epsilon}$ for all $\epsilon>0$. Moreover,

$$
\|u\|_{Z_{\omega}^{s-\epsilon}} \leq C(s, \epsilon)\left(\|u\|_{0, \omega}+B\right) .
$$

The next lemma is straightforward using Theorem 3.2 together with an explicit calculation.

Lemma 3.1. Let $g \in C^{\infty}(\bar{\Lambda})$ be such that $g( \pm 1)=0$, and let $\phi(x)=\frac{g(x)}{\omega(x)}$. The function $\phi$ is in $Z_{\omega}^{s}$ for $0 \leq s<\frac{7}{2}$. If, in addition, $g_{x}(1) \neq 0$ or $g_{x}(-1) \neq 0$, then $\phi \notin Z_{\omega}^{\frac{7}{2}}$.

Next we give the proof of Theorem 3.1.

Proof of Theorem [3.1. The bound $\left\|E_{N}(t) u_{0}\right\| \leq K\left(t, u_{0}\right) N^{-s}$, together with the principle of uniform boundedness, leads to $\left\|E_{N}(t)\right\| \leq K(t) N^{-s}$, for some $K=K(t)$ independent of $N$. Therefore, $\left\|\left(E_{N}(t)\right)^{*}\right\| \leq K(t) N^{-s}$. 
Taking into account that $\left(E_{N}(t)\right)^{*}$ is the error operator associated to problem (51), we have, for any $\phi_{0} \in L^{2}$,

$$
\left\|\phi(t)-\phi^{N}(t)\right\|_{0, \omega} \leq K(t) N^{-s}\left\|\phi_{0}\right\|_{0, \omega},
$$

where $\phi(t)=e^{-t A^{*}} \phi_{0}$ and $\phi^{N}(t)=e^{-t A_{N}^{*}} P_{N} \phi_{0}$.

Then, using Theorem 3.3, we deduce that

$$
\phi(t) \in Z_{\omega}^{s-\epsilon}, \quad \forall \epsilon>0 .
$$

On the other hand, it is clear that $\omega(x) \phi(x, t)$ is the solution of problem (1) with initial condition $\omega \phi_{0}$. Hence $\omega(\cdot) \phi(\cdot, t) \in C^{\infty}(\bar{\Lambda})$ and $(\omega \phi)( \pm 1, t)=0$. Now, if we pick $\phi_{0} \in L_{\omega}^{2}$ such that $(\omega \phi)_{x}( \pm 1, t) \neq 0$, for instance $\phi_{0}(x)=\omega^{-1}(x) \sin \left(\frac{\pi}{2}(x+1)\right)$, Lemma 3.1 gives

$$
\phi(\cdot, t) \in Z_{\omega}^{\frac{7}{2}-\epsilon}, \quad \phi(\cdot, t) \notin Z_{\omega}^{\frac{7}{2}} .
$$

Then, comparing this with (7), we obtain the result.

\section{Some POSITIVE RESUlts}

In this section, we return to the variable coefficient case. We state some error estimates.

\subsection{The Legendre case.}

Theorem 4.1. Let $\omega(x)=1$ and $m \in \mathbb{N}, m \geq 1$, and $l \in \mathbb{N}$. If $a \in C^{m+1}(\bar{\Lambda})$, then there is a constant $C$, depending only on $m, l$ and the function $a(x)$, such that for any $u_{0} \in L_{\omega}^{2}$ and for $t>0$ we have

$$
\left\|\frac{\partial^{l} e^{N}}{\partial t^{l}}(t)\right\|_{\nu, \omega} \leq C N^{-(m+2-\nu)} t^{-\frac{m+2}{2}-l}\left\|u_{0}\right\|_{0, \omega}, \quad \nu=0,1 .
$$

This theorem gives the expected result by analogy with finite elements. The proof is achieved by using the same techniques as for the finite element case, see [10] and 13. We point out that, when obtaining $H^{1}$ estimates in the spectral context, the use of inverse inequalities would result in a loss of optimality. Hence, $H^{1}$ estimates are derived without using inverse inequalities by means of slight modifications of the standard techniques. See [7] for details.

4.2. The Chebyshev case. First we quote a result concerning the coercivity of the bilinear form $a_{\omega}(\cdot, \cdot)$ in the variable coefficient case, whose proof can be found in $[6]$.

Theorem 4.2. Let $\omega(x)=\left(1-x^{2}\right)^{-\frac{1}{2}}$, let $a(x)$ be a function with first order continuous derivative in $\bar{\Lambda}$, and let $a_{\omega}(\cdot, \cdot)$ be defined by (2). There exists a positive constant $\mu_{0}$ such that, if $\mu>\mu_{0}$,

$$
\operatorname{Re} a_{\omega}(v, v)+\mu(v, v)_{0, \omega} \geq \gamma_{\mu}\|v\|_{1, \omega}^{2},
$$

for all $v \in H_{\omega, 0}^{1}$, where $\gamma_{\mu}>0$ is a suitable positive constant depending on $\mu$.

This theorem ensures the well-posedness of the Chebyshev weak formulation of the variable coefficient equation. 
Let $m \in \mathbb{N}$, and assume that the function $a(x)$ is in $C^{m-1}(\bar{\Lambda})$ if $m \geq 2$. We define the space

$$
D_{\omega}^{m}=\left\{v \in H_{\omega}^{m} \mid\left(A^{j} v\right)( \pm 1)=0, \quad 0 \leq j<\frac{m}{2}, \quad j \in \mathbb{Z}\right\} .
$$

Theorem 4.3. Let $m$ be a nonnegative integer, and let us assume that the function $a(x) \in C^{m+1}(\bar{\Lambda})$. Then, for any integer $l \geq 0$ there exists a constant $C$, depending only on $l, m$ and the function $a(x)$, such that for any $u_{0} \in D_{\omega}^{m}$ and $t>0$ the following estimate holds:

$$
\left\|\frac{\partial^{l} e^{N}}{\partial t^{l}}(t)\right\|_{\nu, \omega} \leq C N^{-(m+2-\nu)} t^{-1-l} e^{\mu t}\left\|u_{0}\right\|_{m, \omega}, \quad \nu=0,1 .
$$

This theorem gives essentially a gain of two units in the order of the method with respect to $N$. An arbitrary gain is not possible, due to Theorem 3.1. We also have the following result, which can be considered an improvement of Theorem 4.3 for $m=0$.

Theorem 4.4. Let us assume that $a(x) \in C^{3}(\bar{\Lambda})$. For all integers $l \geq 0$ there exists a constant $C$, depending only on $l$ and on the function $a(x)$, such that for any $u_{0} \in L_{\omega}^{2}$ the following estimate holds for $t>0$ :

$$
\left\|\frac{\partial^{l} e^{N}}{\partial t^{l}}(t)\right\|_{\nu, \omega} \leq C N^{-\frac{5}{2}} t^{-\left(l+\frac{5}{4}+\frac{\nu}{2}\right)} e^{\mu t}\left\|u_{0}\right\|_{0, \omega}, \quad \nu=0,1 .
$$

The proofs of Theorems 4.3 and 4.4 are also similar to analogous results for the finite element case, but here, apart from avoiding the use of "bad" inverse inequalities, some difficulty arises from the fact that $a_{\omega}(\cdot, \cdot)$ is not Hermitian. Hence, some duality arguments involve the study of the Dirichlet problem associated to the operator $A^{*}$, for which there is a lack of regularity. This is overcome by using techniques similar to those in section 4 of [2]. We again refer to [7] for the detailed proofs.

\section{REFERENCES}

[1] J. Bergh and J. Löfström, Interpolation spaces: an introduction, Springer-Verlag, Berlin Heidelberg, 1976. MR 58:2349

[2] C. Bernardi and Y. Maday, Properties of some weighted Sobolev spaces and applications to spectral approximations, SIAM J. Numer. Anal., 26, 1989, 769-829. MR 91c:46046

[3] C. Canuto, M.Y. Hussaini, A. Quarteroni and T.A. Zang, Spectral methods in Fluid Dynamics, Springer-Verlag, New York, 1988. MR 89m:76004

[4] C. Canuto and A. Quarteroni, Spectral and pseudospectral methods for parabolic problems with non periodic boundary conditions, Calcolo, 18, 1981, 197-217. MR 84h:35132

[5] R. Dautray and J. L. Lions, Analyse mathématique et calcul numérique pour les sciences et les techniques, Vol. 3, Masson, Paris, 1985. MR 88i:00003b

[6] J. de Frutos and R. Muñoz Sola, Chebyshev pseudospectral collocation for parabolic problems with nonconstant coefficients, Proceedings of the third international conference on spectral and high order methods, Houston (Texas), 1996, 101-107.

[7] J. de Frutos and R. Muñoz Sola, Error estimates for Galerkin spectral discretizations of parabolic problems with nonsmooth data, Applied Mathematics and Computation Reports 1998/7, Universidad de Valladolid (Spain), pp. 749-754. CMP 98:15

[8] G. Fernández Manín. Algunas contribuciones al estudio del error en los métodos espectrales: optimalidad de los métodos de Jacobi y estudio del método de "patching". PhD Thesis. Santiago de Compostela, 1995.

[9] G. Fernández Manín and R. Muñoz Sola, Polynomial approximation of some singular solutions in weighted Sobolev spaces, Proceedings of the third international conference on spectral and high order methods, Houston (Texas), (1996), 93-99. MR 97g:00019 
[10] H. Fujita and T. Suzuki, Evolution problems, in Handbook of numerical analysis, Vol. II, P. G. Ciarlet and J. L. Lions eds., North Holland, Amsterdam, 1991, pp. 789-928. MR 92f:65001

[11] M. Luskin and R. Rannacher, On the smoothing property of the Galerkin method for parabolic equations, SIAM J. Numer. Anal., 19, 1982, 93-113. MR 83c:65245]

[12] A. Pazy, Semigroups of linear operators and applications to partial differential equations, Springer-Verlag, New York, 1983. MR 85g:47061

[13] V. Thomée, Galerkin finite element methods for parabolic problems, Lecture Notes in Math., vol. 1054, Springer-Verlag, Berlin Heidelberg, 1984. MR 86k:65006

Departamento de Matemática Aplicada y Computación, Universidad de Valladolid, VALLADOLID, SPAIN

E-mail address: frutos@mac.cie.uva.es

Departamento de Matemática Aplicada, Universidad de Santiago de Compostela, Santiago de Compostela, Spain

E-mail address: rafa@zmat.usc.es 